\title{
Acanthamoeba keratitis: multicentre survey in England 1992-6
}

Cherry F Radford, Ordan J Lehmann, John K G Dart, for the National Acanthamoeba
Keratitis Study Group spite recent improvements in diagnosis and treatment, the characteristically recalcitrant pain and protracted clinical course still culminates in severe visual loss for over $15 \%$ of patients. ${ }^{1}$ Although AK can occur after corneal trauma, ${ }^{2}$ particularly in a rural environment, up to $92 \%$ of cases have been associated with contact lens (CL) use. . $^{3-5}$

Although reliable incidence data for AK are not available, in most series the organism has been implicated in less than 5\% of CL related microbial keratitis cases. ${ }^{6-8}$ The incidence in the USA showed a dramatic increase in parallel with the growing popularity of soft CL (SCL) during the 1980 s. $^{4}$ The association with SCL wear was attributed to use of non-sterile CL solutions, swimming while wearing CL, and inadequate disinfection of lenses after use. ${ }^{9}$ Subsequently in the UK a review of 72 cases presenting to Moorfields Eye Hospital between 1984 and 1992 showed a marked rise in the number of cases associated with the introduction and increasing popularity of disposable SCLs. ${ }^{3}$ Further reviews at both Moorfields ${ }^{10}$ and Bristol ${ }^{11}$ confirmed that this trend had continued, at least until mid 1995. A casecontrol study showed that the association with disposable lenses was largely attributable to the increased frequency of omitted or chlorine based disinfection among users of these CLs at the time. ${ }^{12}$

The incidence of $\mathrm{AK}$ appears to be much influenced by changing trends in contact lens care and use. Although the disease is rare, over $7 \%$ of adults in the UK are CL wearers (Eyecare Information Service, London, personal communication, May 1997) and therefore at a greatly increased risk. This multicentre survey was conducted to provide national data on the frequency, outcomes, and associated risk factors for $\mathrm{AK}$ during the 4 year period to September 1996 in England. effective medical therapy have improved the prognosis for most AK patients. The study demonstrates the highly preventable nature of the disease: $91 \%$ of the SCL wearers could have avoided the disease by refraining from inadvisable practices, and a marked fall in frequency was seen after intensive media attention to AK, possibly in conjunction with increasing penetrance of new CL products. Since the frequency of AK appears to be largely determined by the ever changing trends in CL use, continued monitoring is indicated.

(Br f Ophthalmol 1998;82:1387-1392)

Dr C F Radford, Moorfields

Eye Hospital, City Road,

London EC1V 2PD.

Accepted for publication 3 June 1998
Acanthamoeba keratitis $(\mathrm{AK})$ is a rare but potentially devastating corneal infection; de-

\section{Methods}

Data were collected for patients with a diagnosis of AK presenting between 1 October 1992 and 30 September 1996.

For each of the 14 regional health authorities (RHAs) in England a senior ophthalmologist in a large eye unit was invited to act as a regional coordinator for data collection. Regional coordinators organised the completion of clinical data sheets for AK patients in their hospital and sent data sheets to consultants in all other eye departments in their RHA. Patients were usually identified prospectively from late 1995, and retrospectively for earlier presentations. Where necessary, requests for 
Table 1 AK patients identified as presenting 1 October 1992-30 September 1996 in 12 English RHAs (17 overseas patients excluded)

\begin{tabular}{|c|c|c|c|c|c|c|c|}
\hline RHA & $1992-3$ & $1993-4$ & $1994-5$ & $1995-6$ & $\begin{array}{l}\text { Total } \\
\text { cases }\end{array}$ & $\begin{array}{l}\text { RHA population } \\
\text { (from } 1991 \text { census) }\end{array}$ & $\begin{array}{l}\text { Annualised } \\
\text { incidence per } \\
100000\end{array}$ \\
\hline East Anglian & 0 & 0 & 0 & 1 & 1 & 2136023 & 0.0117 \\
\hline Mersey & 0 & 1 & 2 & 2 & 5 & 2400582 & 0.0521 \\
\hline Northern & 1 & 1 & 1 & 2 & 5 & 2986934 & 0.0418 \\
\hline North Western & 4 & 7 & 8 & 0 & 19 & 4006900 & 0.1185 \\
\hline Oxford & 3 & 1 & 2 & 0 & 6 & 2558551 & 0.0586 \\
\hline ^NE Thames & 36 & 39 & 37 & 20 & 132 & 3768783 & 0.8756 \\
\hline SE Thames & 0 & 0 & 3 & 0 & 3 & 3659866 & 0.0205 \\
\hline South Western & 5 & 9 & 9 & 4 & 27 & 3289255 & 0.2052 \\
\hline Trent & 0 & 7 & 2 & 0 & 9 & 4629928 & 0.0486 \\
\hline Wessex & 0 & 1 & 3 & 3 & 7 & 2962329 & 0.0591 \\
\hline West Midlands & 1 & 2 & 4 & 0 & 7 & 5225395 & 0.0335 \\
\hline Yorkshire & 0 & 3 & 2 & 0 & 5 & 3535201 & 0.0354 \\
\hline Total & 50 & 71 & 73 & 32 & 226 & 41159747 & 0.1373 \\
\hline
\end{tabular}

Data were unavailable from North West Thames and South West Thames.

*Includes Moorfields Eye Hospital.

follow up data were made up to 6 months after the end of the study period.

Clinical data sheets were checked for diagnostic criteria. Confirmed cases were defined as patients with a positive culture of Acanthamoeba or a clinical diagnosis which included perineural infiltrates (pathognomonic for the disease $^{3}$ ). Patients with a clinical diagnosis excluding perineural infiltrates but including an array of other typical characteristics - such as positive culture from a CL, CL case or solutions, severe or disproportionate pain, epitheliopathy, limbitis, ring ulcer, and recalcitrant keratitis responding to appropriate antiamoebal therapy - were categorised as having presumed acanthamoeba keratitis.

A postal questionnaire, detailing CL use and care as well as contact with soil, dust, and different types of water, was sent to patients in order to detect possible associations with previously established risk factors. ${ }^{9}{ }^{12}$ These data supplemented the risk factor information supplied by the reporting ophthalmologist on the clinical data sheet. Data were entered on to a computer database.

\section{Results}

DISEASE FREQUENCY

Data were received from 12 of the 14 English RHAs. A total of 243 patients (259 eyes) with a diagnosis of AK were identified among this population of approximately 41 million. The total annualised incidence was 0.14 per 100000 individuals, but there was considerable variation between the 12 participating RHAs (0.01-0.88 per 100 000) (Table 1). At Moorfields Eye Hospital the increase in frequency of cases presenting in the early $1990 \mathrm{~s}^{310}$ (1990, $\mathrm{n}=22 ; 1991, \mathrm{n}=27$ ) has continued, reaching a plateau of approximately 40 cases per year in 1994 and 1995. This has been followed by a dramatic $46 \%$ decrease in the number of cases during the final year of the survey. Although small numbers in the majority of the other RHAs prevent detailed analysis, this pattern appears to have occurred throughout the country (Table 1 ). Seasonal variations in frequency were also identified: a calculation based on Poisson probabilities showed that the observed number of cases was significantly higher than expected for August and Septem- ber and significantly lower than expected for February and March (Table 2).

DIAGNOSIS

A positive tissue diagnosis of Acanthamoeba was made for $131 / 243(54 \%)$ patients, and for a further $53(22 \%)$ diagnosis was confirmed by the presence of perineural infiltrates.

There were 59 cases of presumed AK who had atypical keratitis with an array of characteristic signs and symptoms ${ }^{3}$ such as positive culture from CL paraphernalia accompanying other typical features $(n=48)$, epitheliopathy $(n=27)$, limbitis $(n=19)$, severe or disproportionate pain $(n=17)$, and ring infiltrates $(n=6)$.

The proportion of patients diagnosed early (antiamoebal therapy commenced within 30 days of presentation) was $137 / 200(69 \%)$ during the first 3 years, significantly rising to $33 / 37$ $(89 \%)$ in the final year $\left(\chi^{2}=6.591, \mathrm{p}<0.02\right)$ (Table 3). For six patients insufficient data prevented accurate calculation of diagnostic delay. Among patients who had not been wearing contact lenses only 9/18 (50\%) were diagnosed early.

\section{TREATMENT AND OUTCOME}

For $197 / 218$ patients (90\%) medical therapy included polyhexamethyl biguanide (PHMB) $(168 / 218,77 \%))$ and/or chlorhexidine (44/ $218,20 \%$ ); the most common treatment combination was propamidine isethionate and PHMB (108/218, 50\%) (Table 4). For 25 patients medical therapy details were incomplete. The average duration of medical therapy for patients in the study was 6 months (median 135 days; range 16-886 days), although 26 patients were still on medication or lost to follow up at the time of analysis (6 months after the study period).

Table 2 Seasonal variation in frequency of disease (among UK residents)

\begin{tabular}{lcl}
\hline Month of presentation & New cases & $\begin{array}{l}\text { p Values } \\
\text { (Poisson probabilities) }\end{array}$ \\
\hline January & 18 & 0.484 \\
February & 8 & 0.004 \\
March & 10 & 0.020 \\
April & 16 & 0.305 \\
May & 17 & 0.393 \\
June & 21 & 0.338 \\
July & 25 & 0.100 \\
August & 28 & 0.028 \\
September & 33 & 0.002 \\
October & 24 & 0.142 \\
November & 14 & 0.158 \\
December & 12 & 0.065 \\
Total & 226 & \\
\hline
\end{tabular}

Expected/month $=18.83$.

Table 3 Numbers of patients $(n=237)$ with an early diagnosis in each year of the study (for six patients insufficient data prevented calculation of diagnostic delay)

\begin{tabular}{lll}
\hline & \multicolumn{2}{c}{ Diagnosis } \\
\cline { 2 - 3 } & $\begin{array}{l}\text { Early (<31 } \\
\text { days) } \\
\text { No (\%) }\end{array}$ & $\begin{array}{l}\text { Late (31 days } \\
\text { or more) } \\
\text { No (\%) }\end{array}$ \\
\hline Presentation & $37(71)$ & $15(29)$ \\
\hline 1 Oct 92-30 Sep 93 & $47(64)$ & $26(36)$ \\
1 Oct 93-30 Sep 94 & $53(70)$ & $22(30)$ \\
1 Oct 94-30 Sep 95 & $33(89)$ & $4(11)$ \\
1 Oct 95-30 Sep 96 & $170(72 \%)$ & $67(28 \%)$ \\
\hline
\end{tabular}


Table 4 Antiamoebal treatments received by acanthamoeba keratitis patients in the survey

\begin{tabular}{lc}
\hline Treatments received (in combination or separately) & No (\%) \\
\hline Propamidine + polyhexamethyl biguanide (PHMB) & $108(50)$ \\
Propamidine + neomycin + PHMB & $31(14)$ \\
Propamidine + neomycin & $16(7)$ \\
Propamidine + chlorhexidine & $15(7)$ \\
Propamidine + neomycin + chlorhexidine & $12(6)$ \\
Propamidine + PHMB + chlorhexidine & $8(4)$ \\
Propamidine + neomycin + PHMB + chlorhexidine & $7(3)$ \\
Propamidine + PHMB + topical antifungal & $6(3)$ \\
Propamidine alone & $5(2)$ \\
Propamidine + neomycin + PHMB + topical antifungal & $3(1)$ \\
Propamidine + chlorhexidine + topical antifungal & $1(<1)$ \\
Propamidine + neomycin + chlorhexidine + topical antifungal & $1(<1)$ \\
Propamidine + PHMB + oral antifungal & $1(<1)$ \\
Propamidine + neomycin + PHMB + oral antifungal & $1(<1)$ \\
Propamidine + neomycin + PHMB + topical and oral antifungal & $1(<1)$ \\
Propamidine + neomycin + PHMB + oral antifungal + topical interferon & $1(<1)$ \\
Propamidine + neomycin + PHMB + oral metronidazole & $1(<1)$ \\
Total & $218^{\star}(100)$ \\
\hline
\end{tabular}

${ }^{\star}$ Data were insufficient for 25 patients.

Table 5 Surgery among 243 acanthamoeba keratitis patients (259 eyes) in the survey

\begin{tabular}{lcl}
\hline & No & $\begin{array}{l}\text { (\% of } \\
\text { patients in } \\
\text { survey) }\end{array}$ \\
\hline Surgery & 40 (41 eyes) & $(16)$ \\
$\quad$ Penetrating/lamellar & $30(1$ & $(12)$ \\
$\quad$ keratoplasty & bilateral) & \\
Cataract extractions & 16 & $(7)$ \\
Superficial keratectomy & 7 & $(3)$ \\
Trabeculectomy & 5 & $(2)$ \\
Enucleation & 2 & $(1)$ \\
Discharged, no surgery & 149 & $(61)$ \\
Under review, no surgery yet & 54 & $(22)$ \\
Total & 243 & \\
\hline
\end{tabular}

Surgery was performed on $40 / 243(16 \%)$ patients (41/259 eyes), 30/243 (12\%) patients (31 eyes) receiving penetrating or lamellar keratoplasty (Table 5). No significant difference existed in the proportions of confirmed and presumed $\mathrm{AK}$ cases known to have required surgery $\left(29 / 181\right.$ and $7 / 58$ respectively, $\chi^{2}=$ $0.537)$. Of the 203 patients who had not had any surgery there were 54 who were still under review, 17 of whom still had visual acuities of less than 6/12. Among non-CL wearers (18 unilateral patients) surgery (including two enucleations) was required in 8/18 (44\%) of cases.

The final acuities for eyes of patients who had been discharged $(n=161,175$ eyes) were $6 / 6$ or better for $113 / 175$ (65\%), $6 / 9$ to $6 / 12$ for $53 / 175(30 \%)$, and $6 / 18$ to $6 / 60$ for $5 / 175$ $(3 \%)$. Four patients with unilateral infection were left with light perception $(n=2)$ or anophthalmos $(n=2)$. Among patients still under review ( $n=61,61$ eyes) or lost to follow up $(n=21,23$ eyes) acuities had reached $6 / 6$ or better for $25 / 84(30 \%)$ eyes, $6 / 9$ to $6 / 12$ for $31 / 84(37 \%), 6 / 18$ to $6 / 60$ for $11 / 84(13 \%)$, and unknown for three eyes. Fourteen of 84 eyes had perception of light only. In all, 25/82

Table 6 Distribution of contact lens (CL) types among $A K$ patients who had been using $C L$

\begin{tabular}{lcc}
\hline Contact lens use & No (\%) & $\begin{array}{l}\text { (Estimated \% } \\
\text { of CL users, } \\
\text { sample size }=832)\end{array}$ \\
\hline Soft: disposal frequency $1-4$ weeks & $134(60)$ & $(50)$ \\
Soft: disposal frequency $>4$ weeks or not known & $71(32)$ & $(30)$ \\
Rigid gas permeable & $14(6)$ & $(19)$ \\
Hard & $3(1)$ & $(1)$ \\
Soft-rigid combination & $2(1)$ & None recorded \\
Scleral & $1(<1)$ & None recorded \\
Total & $225(100)$ & $(100)$ \\
\hline
\end{tabular}

(31\%) of these patients were known to be still on medical therapy. When confirmed and presumed AK cases were compared, there was no significant difference in the numbers of patients known to have achieved $6 / 12$ or better $\left(153 / 182\right.$ and 53/58 respectively, $\left.\chi^{2}=1.935\right)$. Although similar numbers in the early and late diagnosed patients achieved at least $6 / 12$, a significantly greater proportion of the early diagnosed group achieved $6 / 6$ or better (99/ 170 compared with $25 / 67, \chi^{2}=8.433, p<0.01$ ). The proportions of patients achieving $6 / 12$ or better among those treated with propamidine and PHMB only (99/107) or propamidine and chlorhexidine only (15/15) were both significantly higher than the proportion of patients with this outcome among those initially treated with propamidine and neomycin sulphate with PHMB added later $(21 / 30)\left(\chi^{2}=10.936\right.$, $\mathrm{p}<0.001$ and 5.625, $\mathrm{p}<0.02$ respectively). Patients who had undergone surgery were significantly less likely to achieve $6 / 12$ or better (23/35) than patients who had received medical therapy only (23/35 compared with 180 / 202, $\left.\chi^{2}=13.287, \mathrm{p}<0.001\right)$.

The visual outcome among non-CL wearers was markedly worse: at the time of analysis (nine were still under review) only 10/18 $(56 \%)$ had achieved $6 / 12$ or better, and six $(33 \%)$ had perception of light or anophthalmos following enucleation.

\section{PATIENT CHARACTERISTICS}

Patient ages ranged from 4 to 64 years, with a mean age of 31.5 years; 135 (56\%) were male and $108(44 \%)$ were female.

\section{RISK FACTORS AMONG NON-CONTACT LENS} WEARERS

Questionnaires were completed by $12 / 18$ (67\%) patients who had not been wearing contact lenses. Eleven $(61 \%)$ of these non-CL wearers had a recent history of trauma (minor in all but three cases) in a garden $(n=4)$, building site $(n=3)$, dairy farming $(n=2)$, fishing $(n=1)$, or nautical $(n=1)$ environment. Four patients had developed the disease following water/snow sporting activities. Data were insufficient for the remaining three patients.

RISK FACTORS AMONG CONTACT LENS WEARERS A total of 225 of the 243 patients $(93 \%)$ had been wearing CLs when their symptoms started, and 157/225 (70\%) completed a questionnaire; $221 / 225$ (98\%) had worn CLs for more than 6 months, and only 5/225 (2\%) CL patients had a medical indication for CL wear (three were aphakic, one was keratoconic, and one had a corneal graft). Data on penetrance of different CL types and modalities are not available for each year of the study period. Recent data on current prescribing habits, ${ }^{13}$ however (which inevitably overestimate the proportion of the population wearing newer CL modalities) suggest that SCLs were overrepresented, and rigid gas permeable lenses markedly underrepresented among the study patients (Table 6).

In contrast with the non-CL users, a minority of the CL wearers had a recent history of 
Table 7 Distribution of types of soft contact lens (SCL) disinfection systems used by AK patients

\begin{tabular}{lc}
\hline SCL disinfection & No (\%) \\
\hline Chlorine release & $83(41)$ \\
Unknown type & $38(19)$ \\
Hydrogen peroxide & $33(16)$ \\
Multipurpose & $8(4)$ \\
Thiomersal and/or chlorhexidine & $5(2)$ \\
Disposal on removal & $3(1)$ \\
None (no lens disposal) & $35(17)$ \\
Total & $205(100)$ \\
\hline
\end{tabular}

trauma $(14 / 225,7 \%)$, and few $(5 / 225,2 \%)$ had acute concurrent ocular disease (two had acute epithelial necrosis after overnight CL use, an aphakic patient with glaucoma had anterior uveitis, one had conjunctivitis unrelated to CL wear, and one had CL related dry eye).

Adequate CL storage case hygiene (weekly rinsing of the case with sterile saline, boiled water, or disinfection solution followed by drying by leaving it open or by wiping with a clean tissue) was carried out by only seven of the 135 (5\%) CL wearers for whom these data were available. Eighty two of 131 storage cases (63\%) tested were culture positive for Acanthamoeba.

Among patients with rigid lenses, 11/17 (65\%) failed to perform regular disinfection, and for three of the four subjects who had been compliant with correct disinfection routines there was a history of swimming pool bathing with contact lenses in situ.

Among SCL wearers for whom disinfection data were sufficient there were 56/150 (37\%) who were disinfecting irregularly and a further $40 / 150(27 \%)$ who failed to carry out any disinfection. Chlorine release systems were overrepresented among the AK patients using soft lenses (Table 7). Fourteen of 158 (9\%) patients (for whom these data were available) were known to have rinsed or soaked their SCL in tap water or other non-sterile water. Forty seven of the 205 (23\%) SCL users were known to have swum or engaged in water sports while wearing their lenses before infection. Among questionnaire respondents who had been wearing SCL, one or more established CL related risk factors ${ }^{9}{ }^{12}$ were identified for 125/138 (91\%) patients (Table 8).

\section{Discussion}

DISEASE FREQUENCY

Despite the wide coverage of this multicentre survey the national annualised incidence of acanthamoeba keratitis may be substantially greater than calculated from the results $(0.14$ per 100 000); regional coordinators could not

Table 8 Frequency of pre-established risk factors for soft contact lens (CL) related acanthamoeba keratitis among questionnaire respondents $(n=138)$

\begin{tabular}{lc}
\hline & No (\%) \\
\hline Swimming with CL in ${ }^{9}:$ & \\
$\quad$ Chlorinated water & $34(25)$ \\
$\quad$ Lakes, rivers, etc & $9(7)$ \\
Sea water & $17(12)$ \\
$\quad$ Any of the above & $47(34)$ \\
Omitted disinfection $^{912}$ (without lens disposal) $^{\text {Chlorine disinfection }}{ }^{12}$ & $85(62)$ \\
Non-sterile (eg tap water, homemade saline) rinsing $^{9}$ & $65(47)$ \\
None of the above & $11(8)$ \\
\end{tabular}

be found for two of the 14 English RHAs, and within each of the contributing RHAs there were units that declined participation or were unable to identify cases retrospectively. In addition, the likelihood of enhanced case detection by larger specialist referral centres, as strongly suggested by the considerably higher incidence rates for North East Thames, North Western, and South Western, prevents any reliable analysis of the geographical distribution of the disease. This large database of cases does, however, enable detection of changing frequency of disease during the 4 year study period, and can show that these changes appear to have occurred throughout the country.

The continued increase in frequency during the $1992-5$ period is likely to be associated with the growing popularity of disposable lenses and the inadequate disinfection routines with which they were associated at the time. ${ }^{12}$ Growing recognition of the disease may also have increased the number of cases diagnosed.

With SCL associated with 205/243 (84\%) of the total number of patients, it is hypothesised that a number of factors improving SCL hygiene practices may have brought about the marked decline in the number of $\mathrm{AK}$ cases during the final year (October 1995-September 1996). During 1993 the first "multipurpose" soft lens disinfection systems were introduced, providing a practical alternative to the chlorine release disinfection systems previously advocated for use with disposable lenses. The latter have been shown, when compared with other systems, to be associated with a 15 times greater risk of developing AK. ${ }^{12}$ The most widely used brands of these new, multipurpose solutions are ineffective against Acanthamoeba. ${ }^{14}$ It is possible, however, that they may provide greater protection from $\mathrm{AK}$ (compared with chlorine release systems) as a result of their surfactant content and recommended "rub and rinse" step, which may be expected to significantly reduce the microbiological load. ${ }^{15}$ These systems also eliminate the need for a separate saline rinsing solution, which, if contaminated or substituted by inappropriate rinsing solution, increases the risk of AK. ${ }^{9}$ The growing popularity of these new solutions, together with the introduction in early 1995 of lenses for disposal after 1 day (thereby obviating the need for lens care solutions), may have contributed to the reduced number of cases in the final year of the study (after autumn 1995). The suddenness of the decline in numbers, however, suggests that the predominant factor may be improved CL hygiene and increased use of more effective disinfection systems after widespread media attention in November 1995 to a CL related AK study publication emphasising the risks associated with chlorine release or omitted disinfection. ${ }^{12}$

As in previous reviews of cases in the USA, $^{516}$ a higher frequency of new cases during the summer/early autumn months was identified. This pattern has been attributed to an increased prevalence of Acanthamoeba in the environment during warmer weather. ${ }^{516}$ An additional association with a summertime flooding of the Iowa River (USA) has also been 
described. ${ }^{16}$ It is also likely that warmer weather greatly increases the number of individuals engaging in water sports and outdoor activities (either in the UK or in much warmer climates), thereby increasing the number of people at increased risk of developing $\mathrm{AK}$ after contact with dust, soil, insect vectors, or environmental water. ${ }^{5}$

\section{TREATMENT AND OUTCOMES}

As in a previous series, ${ }^{3}$ outcomes as measured by final acuity and need for surgery were similar among confirmed and "presumed" cases, validating the inclusion of the latter in the survey.

When comparisons are made with a review ${ }^{3}$ of 72 cases presenting to Moorfields Eye Hospital between 1984 and 1992 a marked improvement in diagnosis and outcome is apparent.

The proportion of patients with an early diagnosis, which, as previously, ${ }^{3}$ is strongly associated with a more successful outcome, increased from $49 \%(35 / 72)$ to $72 \%$ (170/ 237). Possibly mainly as a consequence of this, the percentage of eyes known to receive a corneal graft reduced from $32 \%(23 / 73)$ to $18 \%$ $(31 / 175)$. The percentage of eyes known to have achieved at least $6 / 12$ visual acuity rose from $79 \%(58 / 73)$ to $87 \%(222 / 256)$, even though 84 eyes were still under review (including 26 eyes on medical therapy) at the time of analysis. Increased use of chlorhexidine and (particularly) PHMB, which have been shown to be the most effective agents against Acanthamoeba both in vitro ${ }^{17}$ and clinically, ${ }^{118}$ appears to be another contributing factor.

Failure to show a significantly poorer outcome among patients treated with propamidine and one of the biguanides compared with propamidine and neomycin is probably due to the tendency to introduce a biguanide to failures on the latter regime; patients who received propamidine, neomycin, and subsequent PHMB had a significantly poorer visual outcome compared with those who had received propamidine and a biguanide as the first line of treatment, illustrating the effect of delayed appropriate therapy on the final outcome.

The prognosis for patients contracting the disease in the absence of contact lens wear remains poor. This is probably related to the their frequently more delayed diagnosis. Earlier consideration of $\mathrm{AK}$ as a diagnosis among non-CL wearing patients with atypical keratitis following a history of (usually minor) trauma in a rural, dusty, or watery environment-as described in this study and previously ${ }^{24}$-is advised. It has also been suggested, however, that infection acquired in rural settings may involve a heavier inoculate and/or more virulent organisms, ${ }^{1}$ perhaps leading to more severe disease.

RISK FACTORS AMONG CONTACT LENS WEARERS As in previous series, ${ }^{3-5}$ a very high proportion of patients were CL wearers (93\%), and more than $90 \%$ of these were experienced CL wearers without any other predisposing factors. Rigid lenses were underrepresented in the survey, and all but one case had a history of irregular disinfection and/or swimming pool bathing while wearing contact lenses. Easier removal of Acanthamoeba trophozoites and cysts from the rigid CL surface ${ }^{19}$ may be contributing to the apparently reduced risk with these lenses. The most likely explanation for the apparent difference in risk, however, is that the number of cases using rigid CL has remained fairly static during the 4 year period, while the number associated with SCL rapidly increased during the first 3 years of the survey.

Among normal CL wearers, storage case hygiene is known to be inadequate among $28-86 \%{ }^{20}$ and storage case contamination by Acanthamoeba occurs in up to $7 \% .{ }^{21}$ Perhaps as a result of these universally poor standards, case hygiene has previously failed to be identified as a significant risk factor. ${ }^{12}$ The very high proportion of patients (95\%) in this study failing to carry out the most basic level of case hygiene, together with the frequency with which lens storage cases tested were found to be positive for Acanthamoeba (63\%), however, suggests that increased education regarding this aspect of CL care could further reduce the incidence of $\mathrm{AK}$.

Among SCL wearer questionnaire respondents $(n=138)$ there was a history of at least one previously established risk factor ${ }^{9-12}$ - swimming in lenses (34\%), omitted disinfection $(62 \%)$, chlorine disinfection $(47 \%)$, and non-sterile lens rinsing (8\%)-in $91 \%$ of these patients. These results, together with the high proportion of inadvisable practices among rigid lens wearers, emphasise the highly preventable nature of this disease among contact lens wearers.

\section{Conclusion}

An increased likelihood of earlier diagnosis and the widespread use of more effective medical therapy have greatly improved the prognosis for patients with acanthamoeba keratitis, although a successful outcome for the small proportion of patients with no recent history of CL wear remains less assured. The study demonstrates the highly preventable nature of the disease: $91 \%$ of the soft lens wearers (and probably $94 \%$ of the rigid lens wearers) could have avoided the disease by carrying out more effective disinfection and/or refraining from swimming while wearing lenses, and a dramatic fall in the incidence was seen after intensive media attention prompted widespread discussion and increased education of CL wearers. The gaining penetrance of new CL disinfectants and daily disposable CL may also be contributing factors. There is a continued need to educate CL wearers about these risks, and since the frequency of $\mathrm{AK}$ appears to be largely determined by the ever changing trends in contact lens use, continued monitoring is indicated.

We are grateful for funding from Moorfields Eye Hospital and the Iris Fund for Prevention of Blindness.

National Acanthamoeba Keratitis Study Group participants: Birmingham: PJ McDonnell; Bristol: SD Cook, C Illingworth, S Kilvington; Cambridge: MG Kerr-Muir, J Prydal; East Kilvington; Cambridge: MG Kerr-Muir, J Prydal; East
Grinstead: SM Daya, N Habib; Leeds: B Noble; Liverpool: SB Grinstead: SM Daya, N Habib; Leeds: B Noble; Liverpool: SB
Kaye; London: CF Radford, JKG Dart, M Matheson; 
Manchester: AB Tullo, K Goodall; Nottingham: H Dua, A Sarhan; Oxford: AJ Bron, P Ursell; Southampton: OJ Lehmann; Sunderland: SJ Morgan.

1 Duguid IGM, Morlet N, Allan BDS, et al. Outcome of Acanthamoeba keratitis treated with polyhexamethyl
biguanide and propamidine. Ophthalmology 1997; biguanide and $1587-92$.

2 Sharma S, Srinivasan M, George C. Acanthamoeba keratitis in non-contact lens wearers. Arch Ophthalmol 1990;108: 676-8.

3 Bacon AS, Frazer DG, Dart JKG, et al. A review of 72 cases of Acanthamoeba keratitis 1984-1992. Eye 1993;7: 719-25.

4 Stehr-Green JK, Bailey TM, Visvesvara GS. The epidemiology of Acanthamoeba keratitis in the United States. Am Ophthalmol 1989;107:331-6.

5 Auran JD, Starr MB, Jakobiec FA. Acanthamoeba keratitis. A review of the literature. Cornea 1987;6:2-26.

6 Schein OD, Glynn RJ, Poggio EC, et al. The relative risk of ulcerative keratitis among users of daily-wear and extended-wear soft contact lenses. N Engl F Med 1989;321: 773-8.

7 Cohen EJ, Gonzalez C, Leavitt JG, et al. Corneal ulcers associated with contact lenses including experience with disposable lenses. CLAO 尹 1991;17:173-6.

8 Stapleton F. The pathogenesis and epidemiology of contact lens related disease in cosmetic contact lens wearers. $\mathrm{PhD}$ thesis. London: City University, Department of Optometry and Visual Science, 1991

9 Stehr-Green JK, Bailey TM, Brandt FH, et al. Acanthamoeba keratitis in soft contact lens wearers: a casecontrol study. $\mathcal{F} A M A$ 1987;258:57-60.
10 Dart JKG, Duguid IGM. The epidemiology of Acanthamoeba keratitis in the UK Jerusalem: International thamoeba keratitis in the UK. Jerusalem: Intern
Congress on Ocular Infections, 18-22 July 1995.

11 Illingworth CD, Cook SD, Karabatsas CH, et al. Acanthamoeba keratitis: risk factors and outcome. Br f Ophthalmol 1995;79:1078-82.

12 Radford CF, Bacon AS, Dart JKG, et al. Risk factors for Acanthamoeba keratitis in contact lens wearers: a casecontrol study. BMF 1995;310:1567-70.

13 Morgan P, Ramsdale C, Efron N. Trends in UK contact lens prescribing 1996. Optician 1997;213(5583):35-6.

14 Lowe R. A review of the new multi-function lens care solutions. Optician 1993;207(5454):22-25.

15 Shih KL, Hu J, Sibley MJ. The microbiological benefit of cleaning and rinsing of contact lenses. Int Contact Lens Clin 1985;12:235-42.

16 Lane JA, Mathers WD, Sutphin JE, et al. Variation in cases per month of Acanthamoeba, a waterborne pathogen. Invest Ophthalmol Vis Sci 1997;38:S424.

17 Elder MJ, Kilvington S, Dart JKG. A clinicopathological study of in vitro sensitivity testing and Acanthamoeba keratitis. Invest Ophthalmol Vis Sci 1994;35:1059-64.

18 Hay J, Kirkness C, Seal DV, et al. Drug resistance and Acanthamoeba keratitis: the quest for alternative antiprotozoal therapy. Eye 1994;8:555-63.

19 Kilvington S, Larkin DFP. Acanthamoeba adherence to contact lenses and removal by cleaning agents. Eye 1990;4: 589-93.

20 Claydon BE, Efron N. Non-compliance in contact lens wear. Ophthal Physiol Opt 1994;14:356-64.

21 Devonshire P, Munro FA, Abernathy C, et al. Microbial contamination of contact lens cases in the west of Scotland. Br f Ophthalmol 1993;77:41-5. 